\section{Fatores de risco para a não amamentação: um estudo caso-controle}

\section{Risk factors for not breastfeeding: a case - control study}

Abstract

Objectives: to examine risk factors for not breastfeeding in the State of Pernambuco, in the Northeast region of Brazil, in 2006.

Methods: a case-control study was carried out. with cases (70 children who had never breastfed) and controls (140 children exclusively breastfed for four months or more) were paired by sex and age, based on data from the 3rd State Study of Health and Nutrition, conducted in Pernambuco, in 2006. Both maternal and biological variables relating to the children were examined. Bivariate analyses were used to calculate the crude odds ratio. Adjustments applied using Poisson's logistic regression were employed to selectively incorporate variables with $p<0.20$. Variables were considered to be significant when $p<0.05$.

Results: mothers aged 36 years or older $(p=0.021)$ and lack of guidance with regard to breastfeeding during prenatal care $(p=0.014)$ were risk factors with an odds ratio four to five times higher than others for not breastfeeding.

Conclusions: the two variables shown to be significant suggest that the outlook for controlling not breastfeeding is good. In Pernambuco, the prevalence rate of $5 \%$ for children who have never breastfed is bordering on satisfactory, according to countries with good child health, thereby suggesting that the problem is close to being brought under control.

Key words Breastfeeding, Risk factors, Maternal and child health
Maria de Fátima Costa Caminha 1

Rachel de Sá Barreto Luna Callou Cruz 2

Victor Montenegro Cavalcanti de Acioly 3

Rodrigo Rocha do Nascimento4

Pedro Tadeu Álvares Costa Caminha de Azevedo 5 Pedro Israel Cabral de Lira 6

Malaquias Batista Filho 7

1,2,7 Instituto de Medicina Integral Prof. Fernando Figueira. Rua dos Coelhos, 300. E-mail: fatimacaminha@imip.org.br

3,4,5 Faculdade Pernambucana de Saúde. Recife, PE, Brasil.

6 Universidade Federal de Pernambuco. Recife, PE, Brasil.

\section{Resumo}

Objetivos: analisar os fatores de risco para a não amamentação em Pernambuco, Nordeste do Brasil, em 2006.

Métodos: estudo caso-controle, cujos casos (70 crianças que nunca mamaram) e controles (140 crianças amamentadas exclusivamente por quatro meses ou mais) foram pareados por sexo e idade, tendo como base dados da III Pesquisa Estadual de Saúde e Nutrição realizada em Pernambuco, em 2006. Foram analisados fatores maternos $e$ biológicos das crianças. Calcularam-se, através de análises bivariadas, as razões de chance brutas (odds ratio). Mediante ajustamentos aplicados por regressão logística de Poisson, foram seletivamente incorporadas as variáveis com valor $p<0,20$. Consideraram-se como significativas as variáveis com valor de $p<0,05$.

Resultados: mães com idade igual ou superior a 36 anos $(p=0,021)$ e a falta de orientação sobre o aleitamento no pré-natal $(p=0,014)$ representaram uma razão de chance quatro a cinco vezes mais elevada como fator de risco para a não amamentação.

Conclusões: as duas condições evidenciadas como riscos oferecem boas perspectivas para o controle da não amamentação. Em Pernambuco, a prevalência de $5 \%$ de crianças que nunca mamaram começa a figurar entre os padrões satisfatórios, de acordo com os países com boas condições de saúde da criança, sinalizando que o problema estaria perto de ser controlado.

Palavras-chave Aleitamento materno, Fatores de risco, Saúde materno-infantil 


\section{Introdução}

Apesar de algumas controvérsias no passado, ${ }^{1}$ atualmente está bem estabelecido o consenso de que o leite materno representa a melhor alternativa para a alimentação de crianças nos primeiros meses de vida. Sua composição fisicoquímica (conteúdo ideal de nutrientes, propriedades imunológicas, facilidade de digestão/absorção) propicia efeitos benéficos consideráveis à saúde dos lactentes. Além disso, ressaltam-se vantagens econômicas para a família, benefícios ecológicos para a comunidade 2,3 e melhor desempenho em testes de inteligência na idade adulta, aumentando níveis de escolaridade e renda. 4

O modismo dos leites heterólogos, por força dos interesses econômicos da indústria e do comércio nos primeiros 60 anos do século passado, constituiu um dos maiores e mais prejudiciais desvios em termos de cultura, práticas alimentares e promoção da saúde da história humana, 1 com pesadas consequências para a sobrevivência, desenvolvimento e bem estar de centenas de milhões de crianças em todo o mundo. 2,5

No entanto, apesar do consenso universal sobre as vantagens do aleitamento materno, incluindo a recomendação da Organização Mundial da Saúde (OMS) e Ministério da Saúde, 2-4 bem como os objetivos das políticas públicas, principalmente após a Reunião de Cúpula de Nova York (1990), os avanços obtidos em nível populacional ainda estão bem defasados em relação às metas fixadas.

Assim, em nível mundial, a prática do aleitamento materno exclusivo (AME) em menores de seis meses só foi observada por $39 \%$ das crianças entre 2007 e 2011,6 observando-se que a maioria das crianças nunca mamou ou interrompeu precocemente a amamentação exclusiva.

No Brasil, a duração mediana do AME em 2008 foi de 54,1 dias, depois de evoluir de um patamar de 23,4 dias. ${ }^{7}$ No caso de Pernambuco, avaliações epidemiológicas evidenciaram situações ainda mais preocupantes, desde que, em 1997, apenas $1,9 \%$ das crianças foram mantidas em AME até o sexto mês de vida, evoluindo em 2006 para apenas 8,5\%. ${ }^{8}$ Tratase de um resultado apenas discreto, em relação às metas esperadas.

É evidente que as condições de vida, assistência à saúde, fatores familiares e individuais próprios para cada situação concreta podem atuar, isoladamente ou em conjunto, para determinar a grande variação de desfechos, em termos de duração e tipologias do aleitamento materno. ${ }^{8-14}$ Os resultados discordantes, em escala geográfica (regiões, estados), 13 as tendências temporais e as diferenças entre estratos socioeconômicos constituem um desafio aos formuladores e gestores de programas de saúde e, por extensão, aos estudos sobre o tema.

Dessa forma, o presente estudo objetivou investigar que fatores, ao nível populacional, acham-se associados à ausência de amamentação.

\section{Métodos}

Estudo observacional, tipo caso-controle, aninhado no banco de dados da III Pesquisa Estadual de Saúde e Nutrição (III PESN), ${ }^{15}$ que representou um inquérito de prevalência de base populacional realizado pelo Departamento de Nutrição da Universidade Federal de Pernambuco e pelo Instituto de Medicina Integral Prof. Fernando Figueira, com apoio do Conselho Nacional de Desenvolvimento Científico e Tecnológico.

A coleta de dados da III PESN ocorreu entre maio e outubro de 2006, objetivando atualizar e ampliar dados sobre situação de saúde e nutrição da população de menores de cinco anos do Estado de Pernambuco e respectivas mães e famílias, sequenciando dados obtidos em inquéritos anteriores (I e II PESN) referentes aos anos de 1991 e 1997.16,17 O estudo abrangeu áreas urbanas (Região Metropolitana do Recife e Interior Urbano) e rurais do Estado.

A amostra da III PESN foi calculada com base nas prevalências esperadas de desnutrição energético protéica, anemia, deficiência de vitamina A, diarréia e infecção respiratória aguda (IRA) na população de referência, estimada em 785.522 crianças. ${ }^{17}$ Para esses cinco problemas, considerando suas prevalências e a admissão de erros de estimativas entre 1,5 e $3,0 \%$, resultaram amostras de tamanhos diversos, ou seja, 852 no setor urbano (431 para a Região Metropolitana do Recife, 421 para o interior urbano) e 798 para o setor rural, formando, assim, um consolidado amostral de 1650 crianças.

As informações relativas às crianças foram obtidas por entrevistas junto às mães ou representantes legitimados. Para o grupo de casos, foram excluídas as crianças adotadas, quando a falta de amamentação torna-se autoexplicativa, bem como as menores de três meses de idade, pela impraticabilidade de pareamento com o grupo de controles, fixado, como pré-condição, em pelo menos quatro meses de AME.

Foram consideradas como casos, as crianças que nunca mamaram e como controles, as que foram amamentadas exclusivamente por quatro meses ou mais. O pareamento foi realizado para contornar o efeito da idade e do sexo, dois reconhecidos fatores 
de confundimento dos resultados. Para o pareamento por idade, na proporção de dois controles para cada caso, foram aceitas variações de até um mês para crianças até 36 meses de idade, e de até dois meses para crianças de 37 a 59 meses.

Foi construído seletivamente um banco " $a d$ hoc" de dados, compondo uma amostra de 210 sujeitos, dos quais 70 casos e 140 controles. Atendidas as características de definição, e excetuadas as situações de exclusão, todos os casos do banco de dados se impuseram por si, enquanto os controles se sujeitavam as condições de pareamento.

Definidos os dois grupos, a amostra possibilita um poder de $80 \%$ para detectar um OR (odds ratio) maior ou igual a 3,5 , tendo como exposição não ter tido orientação sobre o aleitamento materno no prénatal e não ter recebido orientação sobre aleitamento materno no pré-natal.

Foram analisadas 16 variáveis, incluindo idade $(<20,20-35,36$ ou mais); anos de estudo $(\leq 4,5-8$, 9 ou mais); renda per capita familiar $(\leq 0,24 \mathrm{SM}$; 0,25-0,49 SM; $\geq 0,5 \mathrm{SM}$ ); área (urbana e rural); zona de moradia (Região Metropolitana de Recife e Interior); disponibilidade de água encanada dentro do domicílio ( $\operatorname{sim}$, não); número de pessoas no domicílio ( 1 a 3,4 ou mais); trabalho materno (sim, não); número de consultas no pré-natal $(\leq 3,4-5,6 \mathrm{e}$ mais); orientações sobre amamentação no pré-natal (sim, não); paridade (primípara e multípara); tipo de parto (vaginal e cesárea); cadastro no Programa Saúde da Família (sim, não); visita do agente comunitário de saúde (sim, não); distância do serviço de saúde de referência básica $(<1 \mathrm{~km}, \geq 1 \mathrm{~km})$; e peso ao nascer da criança $(<2500 \mathrm{~g}, 2500-2999 \mathrm{~g}, \geq 3000$ g).

Admitiu-se nível de significância de 0,05. A proporção esperada de exposição entre os controles foi de 0,10 e o coeficiente de correlação do status de exposição entre os casos e controles pareados de 0,20 . O valor adotado para a proporção esperada de exposição entre os controles foi estabelecido a partir dos dados da pesquisa. Para o coeficiente de correlação do status de exposição entre casos e controles pareados foi adotado o valor sugerido por Dupont. 18 O cálculo do tamanho amostral foi feito utilizandose o software PASS2005.

A análise de dados foi realizada no programa Stata 12.1. Inicialmente foram aplicadas análises bivariadas para identificar possíveis fatores associados ao risco de crianças nunca terem mamado. Foram obtidas as razões de chance brutas (odds ratio) e testadas suas significâncias. Numa segunda etapa foi realizada análise multivariada mediante emprego de regressão logística reunindo as variáveis que, na análise bivariada, apresentaram um valor de $p<20 \%$, com a finalidade de determinar o efeito independente de cada fator de risco identificado na análise bivariada. Para compor o modelo final, após o ajuste estatístico, foram consideradas as variáveis com valor $\mathrm{p}$ menor que $5 \%$.

O estudo foi aprovado pelo Comitê de Ética em Pesquisa do Instituto de Medicina Integral Prof. Fernando Figueira (IMIP), protocolo no 3119-12, em reunião de 12 de setembro de 2012.

\section{Resultados}

Do total de 1650 crianças pesquisadas no inquérito, foram identificadas 81 que nunca mamaram, representando quase $5 \%$ do total de observações da amostra para compor o grupo de casos do estudo. Após seguir os critérios de exclusão, foram retiradas 11 crianças, das quais nove adotivas e duas menores de três meses, restando assim 70 crianças para representar os casos.

Dos 70 casos, 55 mães informaram como principais motivos de não terem amamentado seus filhos: produção insuficiente de leite $(38,2 \%)$, não aceitação por parte da criança $(25,4 \%)$, problemas nas mamas $(14,6 \%)$, decisão materna em não amamentar $(12,7 \%)$, doenças da criança $(7,3 \%)$ e por fím, motivos de trabalho/estudo materno $(1,8 \%)$.

$\mathrm{Na}$ Tabela 1 são apresentadas as características da amostra estudada e as análises estatísticas dos possíveis fatores de risco para os casos, crianças que nunca foram amamentadas. A idade materna predominante foi de 20-35 anos, com renda igual ou menor a 0,24 salário mínimo, residiam no interior do Estado, com serviço de água encanada, as mães não trabalhavam, haviam recebido seis ou mais consultas pré-natal, incluindo orientação sobre o aleitamento materno. Ademais, a maior parte era constituída de multíparas, tiveram parto vaginal, eram cadastradas no Programa Saúde da Família e tiveram crianças com peso ao nascimento $>3000 \mathrm{~g}$.

Verifica-se que nas análises bivariadas que as variáveis idade da mãe (36 anos ou mais), o espaço geográfico (interior do Estado e área rural), a não disponibilidade de água encanada dentro da residência, a falta de orientação sobre o aleitamento materno na assistência pré-natal, a paridade e o baixo peso ao nascer $(<2500$ gramas $)$ estiveram estatisticamente associadas ao evento a um nível de significância de $p<0,20$, satisfazendo, assim, o critério de inclusão no modelo de regressão logística multivariada. Permaneceram no modelo final a idade materna de 36 anos ou mais $(p=0,021)$ e a falta de orientação sobre o aleitamento materno na assistência pré-natal $(p=0,014)$. Nestas duas condições, o risco de a criança nunca ter sido 
amamentada foi $5,0(1,6-15,8)$ e $3,9(1,3-11,8)$ vezes

maior respectivamente.

\section{Tabela 1}

Características da população do estudo e fatores associados ao risco de crianças menores de cinco anos nunca terem sido amamentadas no Estado de Pernambuco, 2006.

\begin{tabular}{|c|c|c|c|c|c|c|c|c|c|c|}
\hline \multirow[t]{2}{*}{ Variáveis } & \multicolumn{2}{|c|}{ Casos } & \multicolumn{2}{|c|}{ Controles } & \multirow[t]{2}{*}{ OR $_{\text {bruta }}$} & \multirow[t]{2}{*}{ IC95\% } & \multirow[t]{2}{*}{$p$} & \multirow[t]{2}{*}{$\mathrm{OR}_{\text {ajustada }}$} & \multirow[t]{2}{*}{ IC95\% } & \multirow[t]{2}{*}{$p$} \\
\hline & $n$ * & $\%$ & $n^{*}$ & $\%$ & & & & & & \\
\hline \multicolumn{11}{|l|}{ Socioeconômicas e demográficas } \\
\hline Idade das mães (anos) & & & & & & & $<0,001$ & & & 0,021 \\
\hline$<20$ & 7 & 10,0 & 13 & 9,3 & 2,3 & $(0,8-6,9)$ & & 1,9 & $(0,5-7,6)$ & \\
\hline $20-35$ & 36 & 51,4 & 115 & 82,1 & 1 & & & 1 & & \\
\hline 36 ou mais & 27 & 38,6 & 12 & 8,6 & 6,5 & $(2,9-14,7)$ & & 5,0 & $(1,6-15,8)$ & \\
\hline Anos de estudo das mães & & & & & & & 0,519 & & & \\
\hline$\leq 4$ & 29 & 42,0 & 48 & 34,3 & 1,5 & $(0,7-3,1)$ & & & & \\
\hline $5-8$ & 26 & 37,7 & 54 & 38,6 & 1,2 & $(0,6-2,7)$ & & & & \\
\hline 9 ou mais & 14 & 20,3 & 38 & 27,1 & 1 & & & & & \\
\hline $\begin{array}{l}\text { Renda per capita } \\
\text { familiar }(\mathrm{SM})^{\star *}\end{array}$ & & & & & & & 0,355 & - & - & \\
\hline$\leq 0,24$ & 29 & 42,1 & 69 & 49,3 & 1 & & & & & \\
\hline $0,25-0,49$ & 27 & 39,1 & 41 & 29,3 & 1,6 & $(0,8-3,0)$ & & & & \\
\hline$\geq 0,5$ & 13 & 18,8 & 30 & 21,4 & 1,0 & $(0,4-2,3)$ & & & & \\
\hline Área & & & & & & & 0,082 & & & 0,528 \\
\hline Urbana & 33 & 47,1 & 83 & 59,3 & 1 & & & 1 & & \\
\hline Rural & 37 & 52,9 & 57 & 40,7 & 1,7 & $(0,9-3,2)$ & & 1,4 & $(0,5-4,4)$ & \\
\hline Zona & & & & & & & 0,094 & & & 0,291 \\
\hline RMRa & 17 & 24,3 & 50 & 35,7 & 1 & & & 1 & & \\
\hline Interior & 53 & 75,7 & 90 & 64,3 & 1,7 & $(0,9-3,2)$ & & 1,6 & $(0,6-4,0)$ & \\
\hline Água encanada/tratada & & & & & & & 0,166 & & & \\
\hline Sim & 38 & 54,3 & 90 & 64,3 & 1 & & & 1 & & 0,790 \\
\hline Não & 32 & 45,7 & 50 & 35,7 & 1,5 & $(0,8-2,7)$ & & 1,2 & $(0,4-3,5)$ & \\
\hline No de pessoas no domicílio & & & & & & & 0,383 & & & \\
\hline 1 a 3 & 11 & 15,7 & 29 & 20,7 & 0,7 & $(0,3-1,5)$ & & & & \\
\hline 4 ou mais & 59 & 84,3 & 111 & 79,3 & 1 & & & & & \\
\hline Trabalho & & & & & & & 0,426 & & & \\
\hline Sim & 26 & 37,1 & 60 & 42,9 & 0,8 & $(0,4-1,4)$ & & & & \\
\hline Não & 44 & 62,9 & 80 & 57,1 & 1 & & & & & \\
\hline \multicolumn{11}{|c|}{ Obstétricas e assistência pré-natal } \\
\hline Consultas PNb & & & & & & & 0,392 & & & \\
\hline$\leq 3$ & 5 & 9,3 & 8 & 6,1 & 2,5 & $(0,6-9,5)$ & & & & \\
\hline $4-5$ & 12 & 22,2 & 29 & 22,1 & 0,9 & $(0,4-2,2)$ & & & & \\
\hline 6 e mais & 37 & 68,5 & 94 & 71,8 & 1 & & & & & \\
\hline $\begin{array}{l}\text { Orientações amamentação } \\
\text { no PNb }\end{array}$ & & & & & & & 0,002 & & & 0,014 \\
\hline Sim & 40 & 72,7 & 126 & 92,7 & 1 & & & 1 & & \\
\hline Não & 15 & 27,3 & 10 & 7,3 & 3,9 & $(1,6-9,5)$ & & 3,9 & $(1,3-11,8)$ & \\
\hline Paridade & & & & & & & 0,119 & & & 0,183 \\
\hline Primípara & 17 & 27,9 & 23 & 16,7 & 1,7 & $(0,9-3,6)$ & & 1,8 & $(0,7-4,6)$ & \\
\hline Multípara & 44 & 72,1 & 115 & 83,3 & 1 & & & 1 & & \\
\hline
\end{tabular}

Fonte: III Pesquisa Nacional em Saúde e Nutrição no Estado de Pernambuco, 2006.

a Região Metropolitana do Recife; b Pré-natal; c Programa de Saúde da Família; d Agente Comunitário de Saúde. 
Características da população do estudo e fatores associados ao risco de crianças menores de cinco anos nunca terem sido amamentadas no Estado de Pernambuco, 2006.

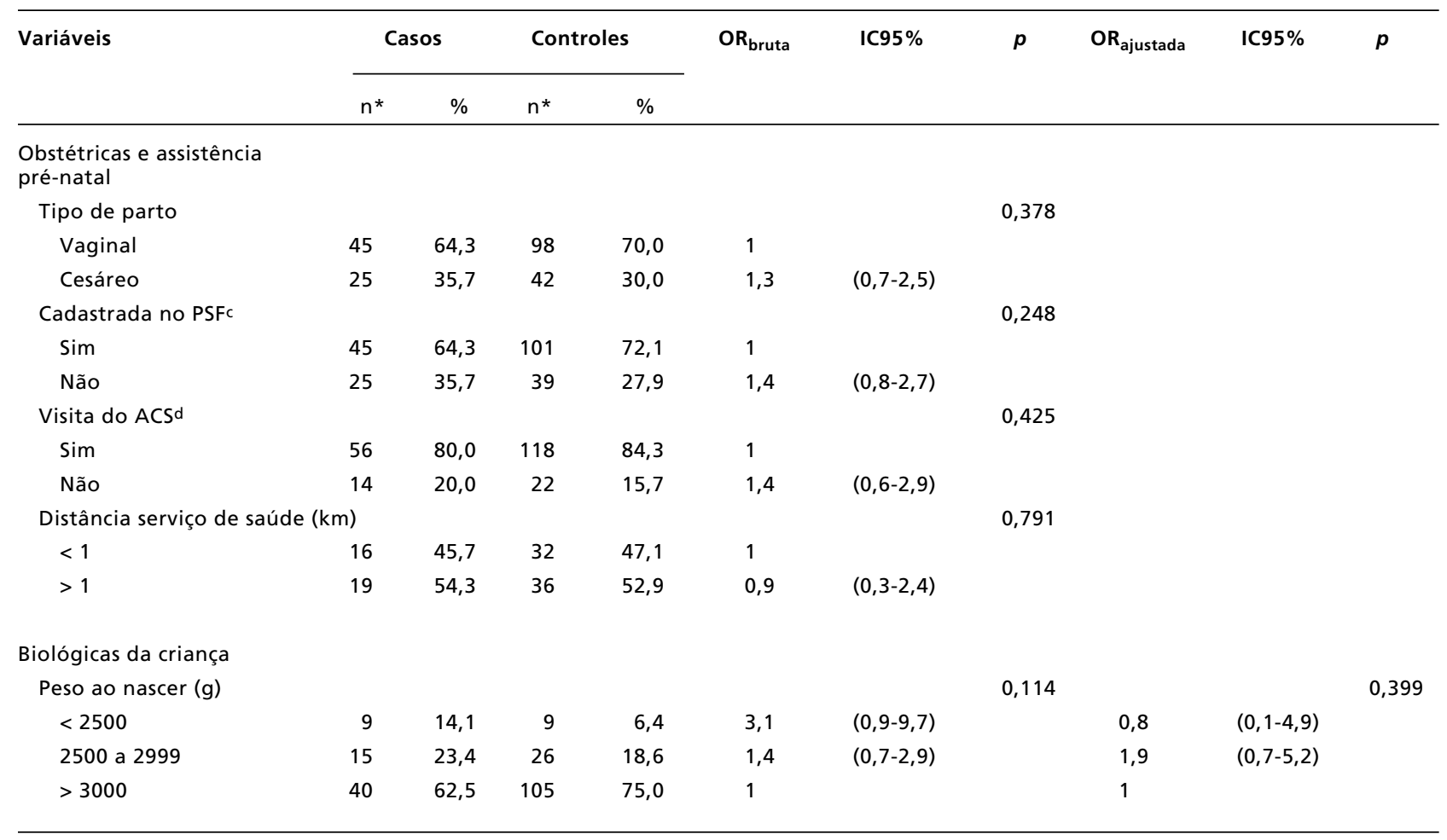

Fonte: III Pesquisa Nacional em Saúde e Nutrição no Estado de Pernambuco, 2006.

* Variações da amostra em cada categoria se devem a eventual falta de informações; * Salário mínimo;

a Região Metropolitana do Recife; b Pré-natal; c Programa de Saúde da Família; d Agente Comunitário de Saúde.

\section{Discussão}

Partindo de uma prática instintiva, o aleitamento materno tornou-se um evento essencialmente comportamental na história recente da humanidade, vinculando-se às influências de muitos fatores que reúnem condicionamentos ambientais, econômicos (sobretudo mercadológicos), políticos, ideológicos, éticos e até implicações ecológicas. ${ }^{19,20}$ Constitui assim um processo complexo na sua determinação e suas consequências no espaço saúde/doença e que se aplica praticamente a todo o ciclo vital do homem.2,4

No caso particular do estado de Pernambuco configura-se, no estágio atual das grandes mudanças que caracterizam o rápido processo de transição epidemiológica, uma situação de evidente polarização dos resultados. Isto se caracterizaria, como fato positivo, pela ocorrência já baixa (cerca de 5\%) de história de falta completa de amamentação, contrapondo-se à frequência também ainda muita baixa de AME, ou seja, 8,1\%,15 como fator negativo.

Esta conjuntura de resultados a partir de amostras probabilísticas de estudos sobre o estado de saúde e nutrição materno infantil em Pernambuco15-17 oportuniza e justifica o estudo caso/controle aqui relatado. Ou seja, o que pode valer como fatores de diferenciação de riscos numa situação desfavorável que deve ser controlada (a ausência completa de amamentação) em contraponto à meta internacional de que todas as crianças possam ser mantidas em AME nos primeiros seis meses de vida. 5,6 De fato, o que aqui se analisa, como controle, são crianças que mamaram exclusivamente durante quatro meses de vida, face à impossibilidade de se contar com um número estatisticamente consistente de observações para representar a condição AME nos seis meses iniciais de vida, como seria ideal.

O conjunto de variáveis estudadas, representando 16 grupos de fatores, constitui a base informativa de inquéritos seguidamente aplicados desde 1991. Na realidade, os instrumentos de coleta de dados, compreendendo variáveis explanatórias e desfechos, vêm sendo diversificados na sequência temporal 1991, 1997 e 2006, representando, por ordem cronológica, a I, II e III PESN. Vale para 
representar diferentes aspectos, como o consumo alimentar (sobretudo de crianças), incluindo o aleitamento materno e a condição nutricional de menores de cinco anos, como estado de nutrição energético proteica, anemia e deficiência de vitamina A. São, portanto, muitos fatores e diversos desfechos, o que representa uma limitação preliminar para o estudo.

É evidente que para todos esses desfechos, incluindo a prática de amamentação das crianças, a relação entre variáveis independentes e respostas analisadas não é a mesma, daí o trabalho de seleção, a partir do banco geral de dados, de fatores que interessam mais de perto a questão da amamentação. Neste sentido, a constatação de que a escolaridade materna, a renda per capita familiar, o macroambiente (espaços geográficos) e microambiente (tipo de moradia e condições de saneamento) e ocupação da mãe já não figuram como antecedentes de discriminação de risco entre os casos e controles.

Trata-se, de fato, de uma nova situação, implicando na mudança dos percentuais de prevalência de crianças jamais amamentadas, afortunadamente agora bem baixa, e, sobretudo, influindo nas mudanças dos fatores de risco, agora restritos à idade das mães e à falta de orientação sobre a amamentação na vigência do pré-natal. São, de fato, comportamentos bem distintos, de vez que, por evidências apoiadas em estudos anteriores, $21-23$ era de se prever que pelo menos a baixa escolaridade, o parto cirúrgico, a baixa faixa etária das mães, a inadequação da assistência pré-natal, a primiparidade e a falta de acesso à Estratégia de Saúde da Família (ESF) pudessem atuar como fatores adversos para a prática saudável da amamentação.

Cabe considerar que a prevalência de $5 \%$ de crianças que nunca foram amamentadas já começa a figurar entre as estatísticas satisfatórias dos padrões de ocorrência de países com boas condições de saúde da população pediátrica. ${ }^{5} \mathrm{Ou}$ seja, o problema estaria perto de ser controlado. O contrário acontece com a situação de onde foram retirados os controles, desde que, de toda a amostra de crianças avaliadas em Pernambuco, apenas $15 \%$ foram amamentadas por pelo menos quatro meses de idade, quando se esperaria, em termos ideais, que todas se mantivessem em regime de AME até seis meses. Desta forma, como questão de saúde, a magnitude do problema aparece mais nos controles do que nos casos, constituindo uma nova situação a ser considerada em futuros estudos sobre o tema.

Por outro lado, a identificação de dois grupos de fatores que se mantiveram no modelo ajustado (ou final) de análises multivariadas (a idade das mães acima de 35 anos e a ausência de ações educativas em favor do aleitamento materno durante a assistência pré-natal) pode ser indicativa de duas condições que devem ser valorizadas. Assim, mães com 36 ou mais anos pertenceriam a uma geração de certa forma "refratária" às práticas de aleitamento materno, representando uma conduta que começa a desaparecer nas gerações mais novas. Estas mães, apresentando um risco relativo elevado $(\mathrm{OR}=5,0 \mathrm{na}$ análise ajustada) expressariam, por sua baixa representação populacional, um risco atribuível baixo, no conjunto de resultados. Já em relação ao papel positivo da orientação educativa do pré-natal para reduzir o risco de ausência de amamentação no histórico alimentar das crianças, passa a configurar uma observação muito interessante para o resgate da prática da amamentação, segundo os padrões recomendados. Esta é, por sinal, uma condição que começa a aparecer em estudos mais recentes, em Pernambuco14-17 e no Brasil.14,23 Considerando conjuntamente as duas observações e a projeção de suas tendências, uma perspectiva bem promissora para os próximos anos, no que se refere às práticas corretas de amamentação.

É possível, ainda, conjecturar que o elenco de variáveis aqui consideradas no exercício analítico deixe de relacionar outras informações possivelmente mais específicas e mais pertinentes para explicar as práticas mais atuais de aleitamento materno. Sem excluir esta possibilidade, vale a pena referir que a fidelidade a um questionário que vem sendo aplicado em três estudos (1991,1997 e 2006) torna-se uma condição básica para se avaliar tendências temporais. Claro que isto não estabelece uma condição imutável e a própria repetição de novos inquéritos populacionais, como será realizado no corrente ano, justificaria as futuras avaliações e suas eventuais necessidades de mudanças.

\section{Agradecimentos}

Ao Instituto de Medicina Integral Prof. Fernando Figueira - IMIP

Ao Conselho Nacional de Desenvolvimento Científico e Tecnológico - CNPq

Ao Departamento de Nutrição da Universidade Federal de Pernambuco - DN/UFPE

Ao Programa de Iniciação Científica pelo IMIP com apoio do $\mathrm{CNPq}$

A Faculdade Pernambucana de Saúde - FPS. 


\section{Referências}

1. Caminha MFC, Serva VB, Arruda IKG, Batista Filho M Aspectos históricos, científicos, socioeconômicos e institucionais do aleitamento materno. Rev Bras Saude Mater Infant. 2010; 10 (1): 25-37.

2. Li R, Dee D, Li CM, Hoffman HJ, Grummer-Strawn LM Breastfeeding and risk of Infections at 6 years. Pediatrics. 2014; 134 (Supl.1): S13-20.

3. Heikkilä K, Kelly Y, Renfrew MJ, Sacker A, Quigley MA. Breastfeeding and educational achievement at age 5 . Matern Child Nutr. 2014; 10 (1): 92-101.

4. Victora CG, Horta BL, de Mola CL, Quevedo L, Pinheiro RT, Gigante DP, Gonçalves H, Barros FC. Associação entre aleitamento materno e inteligência, nível de escolaridade e renda aos 30 anos de idade: um estudo prospectivo de coorte de nascimento do Brasil. Lancet. 2015; 3 (4): 199205.

5. WHO Collaborative Study Team on the Role of Breastfeeding on the Prevention of Infant Mortality. Effect of breastfeeding on infant and child mortality due to infectious diseases in less developed countries: a pooled analysis. Lancet. 2000; 355 (9209): 1104.

6. UNICEF (Fundo das Nações Unidas para a Infância). Situação Mundial da Infância, 2013: Crianças com Deficiência. Brasília; 2013. [Internet]. [acesso em 1 mar 2014]. Disponível em: http://www.unicef.org/brazil/pt/ PT_SOWC2013.pdf

7. Venâncio SI, Escuder MM, Saldivas SRDM, Giugliani ERJ. A Prática do aleitamento materno nas capitais brasileiras e Distrito Federal: Situação atual e avanços. J Pediatr. 2010; 86 (4): 317-24.

8. Caminha MFC, Batista Filho M, Serva VB, Arruda IKG, Figueiroa JN, Lira PIC. Tendências temporais e fatores associados à duração do aleitamento materno em Pernambuco. Rev Saúde Pública. 2010; 44 (2): 240-8.

9. Morais AMB, Machado MMT, Aquino PS, Almeida MI. Vivência da amamentação por trabalhadoras de uma indústria têxtil do Estado do Ceará, Brasil. Rev Bras Enferm. 2011; 64 (1): 66-71.

10. Hung KJ, Berg O. Early skin-to-skin after cesarean to improve breastfeeding. MCN Am J Matern Child Nurs. 2011; 36 (5): 318-24.

11. Machuca MS, Quadros AP, Castro JC, Siqueira GD, Venturi I. Dificuldades encontradas pelas puérperas em relação ao aleitamento materno no pós-parto. Pediatr Mod. 2011; 47 (4): 134-8.

12. Wenzel D, Souza SB. Prevalência do aleitamento materno no Brasil segundo condições socioeconômicas e demográficas. Rev Bras Cresc Desenvolv Hum. 2011; 21 (2): 251-8.
13. Souza SNDH, Migoto MT, Rosetto EG, Mello DF. Prevalence of breastfeeding and associated factors in the municipality of Londrina (PR, Brazil). Acta Paul Enferm. 2012; 25 (1): 29-35.

14. Oliveira MGOA, Lira PIC, Batista Filho M, Lima MC. Fatores associados ao aleitamento materno em dois municípios com baixo índice de desenvolvimento humano no Nordeste do Brasil. Rev Bras Epidemiol. 2013; 16 (1): 17889

15. III Pesquisa Estadual de Saúde e Nutrição - Pernambuco, 2006 DN/UFPE IMIP SES/PE. Situação Alimentar, Nutricional e de Saúde no Estado de Pernambuco: Contexto Socioeconômico e de Serviços. Síntese dos Resultados. Pernambuco, 2008. [Internet]. [acesso em 20 março 2014]. http://pesnpe2006.blogspot.com.br/.

16. Pernambuco. Governo do Estado. Crianças e adolescentes em Pernambuco: saúde, educação e trabalho. Recife: Unicef; 1992

17. Pernambuco. Governo do Estado. II Pesquisa Estadual de Saúde e Nutrição: Saúde, Nutrição, Alimentação e Condições Sócio-econômicas no Estado de Pernambuco. Recife; 1998.

18. Dupont WD. Power calculations for matched case-control studies. Biometrics. 1988; 44 (4): 1157-68.

19. Jones JR, Kogan MD, Singh GK, Dee DL, GrummerStrawn LM. Factors associated with exclusive breastfeeding in the United States. Pediatrics. 2011; 128 (6): 1117-25.

20. Saldiva SRDM, Venancio SI, Gouveia AGC, Castro ALS, Escuder MML, Giugliani ERJ. Influência regional no consumo precoce de alimentos diferentes do leite materno em menores de seis meses residentes nas capitais brasileiras e Distrito Federal. Cad Saúde Pública. 2011; 27 (11): 225362

21. Damião JJ. Influência da escolaridade e do trabalho maternos no aleitamento materno exclusivo. Rev Bras Epidemiol. 2008; 11 (3): 442-52.

22. Gigante DP, Victora CG, Barros FC. Nutrição materna e duração da amamentação em uma coorte de nascimento de Pelotas, RS. Rev Saúde Pública. 2000; 34 (3): 259-65.

23. Vieira GO, Almeida JAG, Silva LR, Cabral VA, Santana Netto PV. Fatores associados ao aleitamento materno e desmame em Feira de Santana, Bahia. Rev Bra Saude Mater Infant. 2004; 4 (2): 143-150.

Recebido em 31 de julho de 2014

Versão final apresentada em 2 de março de 2015

Aprovado em 1 de abril de 2015 